\title{
Community Networking as Radical Practice
}

\author{
Garth Graham
}

$<$ garth.graham@,telus.net >

\begin{abstract}
The practitioners of community networking sense that its nature as a "Movement" is ending. But what does that mean? As daily life online becomes ordinary, and as capacity to engender community online becomes an ordinary function of daily life, what is effective in advancing community networking as a community of practice? How can we get a clear view of community networking as radical practice in social change? Finding answers to these questions requires, in part, defining the roles of technologies and individuals in the structuring of social networks in unexpected ways. Effective community networking then occurs when individuals act cooperatively to make the Internet "happen" more rapidly in support of the need for community and the need for change in local governance.
\end{abstract}

\section{Introduction}

In a way, this essay is going to be about the end of the community networking movement, but it's more about the start of something else. In a networked world, where the connections we have to each other are infinitely reconfigurable, how can someone who is committed to social action align themselves with an emerging and radically different form of practice?

Recently, I poked around on the web to check up on the status of "Community Networking Guides." Here's the pattern I found:

- All of the so-called guides assume that a dense structure of ICTs is already in place or accessible. They are not useful in the context of starting from zero in a "digital divide."

- In essence, people stopped writing community networking guides in approximately 2001. Most of them are more "historical" than useful, and the majority of urls that my search revealed were inactive.

At their last global meeting in Montreal, October 2002, the members of the Global Community Networking Partnership (GCNP) did not come to any agreement on how to represent the community networking movement at the World Summit on the Information Society (WSIS). In follow-up reflections on the consequences of that disengagement, one person on a GCNP list concluded, "The real issue in GCNP, I feel, is not so much about whether an organization exists or not, but whether enough of us share the same political and social vision to take it forward." Looking back now, it is apparent we did not.

This essay grew out of an earlier paper that attempted to explore what we knew about the practices of community networking and its relationship to those defining qualities of what I am going to call an Informed Society that the WSIS process has not and does not address. From the perspective of community networking as a practice, all members of the drafting team for that earlier paper had concluded, "the core WSIS process is a waste of time." 
But then, in January 2004, a split occurred in the discussion of what that conclusion meant. Some of the team were not convinced that GCNP itself was all that relevant to the essential questions posed by the practices of community networking either. But we all did agree with the concept that community as it moves online becomes the critical structural element of an Informed Society. We believe that development and community development have become synonymous.

The question then became - now that we have moved into that Society and community online is evident in many aspects of daily life, what is effective in advancing community networking as a community of practice?

I shelved that earlier paper while I thought about this. That thinking has now stretched over more than a year. What time and distance now allows me to see more clearly is that GCNP is dead, not just dormant. Anyone attempting to revive it under that name would really be starting from zero. Why did GCNP not surface in the first round of WSIS? Because it no longer existed. But that begs the question - what caused it to disappear?

I have been an actor, more than minor although somewhat less than important, in the dialogue to define community networking as a movement on a world stage. For me personally, as that movement fades there is an essence of belonging that also disappears. At first, I felt that disappearance as a puzzle and a loss. But I have come to accept it. The end of community networking as a movement is not the end of community online. Anything but!

Here is an example of what I mean. Somewhere among the mountains of British Columbia, in one of those places where the rivers all run God knows where, there is a community that owns its own broadband telecommunications backbone. I asked them, "How did you do that? Why does it work so well?" Their answer was, "We just practice the politics of small minds."

This essay then is not a lament. It is about acceptance of a change. And, since community online continues everywhere to flow into the nooks and crannies of daily life, this essay has to be about the politics of small minds.

\section{Technology is the way things are done}

To get to a clear view of community networking as radical practice in social change, first we have to put the damn technologies in their place. Here are two of my key assumptions about what the use of technology actually means:

a. According to Ursula Franklin, technology is the "the way we do things around here." This is a process definition - relating technology to practice rather than to objects, products or tools. I think it's reasonable to modify her definition to include "the way we see things around here."

b. Internet Protocol (IP) is a symptom of a shift in emergent global culture toward the autonomy of individuals to define themselves. It is not a cause of that shift. It is a change in the way of seeing that has consequences for how technologies are expressed. And, although that change in perception first surfaces in European arts and science at the end of the 19th Century, it is not particularly Western.

I have tested these assumptions by experience - in the sense of T.S. Eliot's "these fragments I have shored against my ruin." When you see IP as symptom, you see cultural transformation in a particular way. That view has certain useful consequences for the ways in which you can approach practice. You can:

- Completely bypass the kind of technological determinism that sees the tools themselves as the cause of transformation.

- See the "uses" half of the question of the uses of ICTs for development in the context of acculturation processes (remembering that two cultures encountering each other modify each other in both directions).

- Look at what people actually do in daily life online as the path to a deeper understanding of transformative processes and social change.

- See that the drive to individual autonomy through self-definition creates a conflict between open systems that learn and closed systems that are taught.

- See indigenous knowledge (what our system here knows) as the critical element in the capacity for self-definition and self-organization. 
- Accept that seeking neutrality or objectivity will rapidly take you into the absurd. You are either online in the system, or you are not. In participant observation, if you define yourself as an observer, then you must realize that the system must define you as a problem to be learned around.

- It lets you examine that word "global" in a particular way. If by global you make whole/part assumptions about the structure of society, then you are not being fractal enough to encompass the structural implications of distributed systems. In permeable dynamic networks, there is no centre. And if you inhibit the capacity of any to connect to any, you won't stay open enough for the "edges" to get smart. (The distribution of nodes will, however, follow a power law. But that's another story.)

There are three significant and converging changes in the way things are done that have social consequences we can only dimly anticipate:

- Internet Protocol (IP) is the harbinger of a radical shift in the nature of structure toward selforganization.

- Within that altered structure, the human-machine interface is evolving to a point where many of the machines can act as autonomous social agents.

- In the context of rapidly evolving groupwares, all social agents--human or machine--are steadily increasing their capacity to alter the context of social interaction at will by simulating the real world of experience.

In effect, in the Industrial Society, we externalized our hands. In the Informed Society, we are in the process of externalizing our imaginations. If society depends on the way we see it, and the way we see it is infinitely re-configurable, not only within the limits of human imagination but in concert with machines that anticipate behaviour acting as autonomous agents, then the default setting on reality becomes optional.

\section{Rebel administration}

To get to a clear view of community networking as radical practice in social change, next we have to think about the nature of what opposes us. Some communities of practice have more trouble adjusting to the Informed Society than others

Once upon a time, a wise person told me that community was the antithesis of public administration, and that I should not give the managers of technocracy a road map to oppose the consequences of taking community online. In my passion to understand, and to share that understanding, I suspect that I have sometimes ignored that very good advice. But I don't think advice about hiding the road maps matters too much longer.

It seems as if the sleep of managers in public sector administration is over. Some of them at least can now be seen sending their mapmakers out into that previously unknown and unsuspected territory that they call e-governance and I am calling the Informed Society. When the mapmakers return (the ones who don't go native), the administrative immune system still eats most of them. But not all. For an example of extreme change in the technocrats' changing view of the way things are done, Google the military's use of the concept "Network Centric Warfare."

Most public policies for addressing "digital divides" continue to define the relation between community online and the internet as merely an issue in the introduction of technologies in the management of institutions, a sort of bringing of digital civilization to the unelectronic. They assume the technologies in isolation, and then ask, in effect, "Is an institutional response to them called 'community networking' sustainable? Or, are there other forms of institutional or market-based response to the "problem" of introducing technologies at the community level that have greater utility and effectiveness in achieving goals for bridging digital divides?" But, because the question you ask will shape the answer you get, continuing to address only the introduction of ICTs seems precisely the wrong place to start.

A digital strategy for governance online cannot function if its implementers imagine that some sort of separation exists between suppliers of government as services and consumers of those services. When all actors are of equal value in sustaining the dynamics of open systems then anyone who interacts (anyone who speaks) can alter the structure of interaction. Unless that can happen, the systems become static (i.e. they die). In such "structures," (in such communities) the solutions will only emerge from the edges, never 
from the centre. After all, isn't that what TCP/IP was designed to do, and will continue to do until governments destroy it, push the smarts to the edges?

To put that another way, both the Emperor and the little boy who criticized his nakedness are equally capable of creating a circumstance in which the Empire will learn.

Community networking, on the other hand, assumes a set of social and cultural changes of which the Internet is a symptom and then asks: "What new social forms are the most viable in that altered environment?" Community networking's answer is that the form or process called "community," re-defined by being online, is the one most viable. In that view then, community networking is not defined institutionally but as the shared experiences of communities of practice related to understanding how community is achieved in the online context as a public good and an essential socio-economic goal.

The public in highly connected societies, and even some of their politicians, do understand the need for community-based approaches to ICT use as an issue of the public good. The obstacle for the inclusion of that concept in public policy must reside in the intransigence of management in public sector administration. Since now even most public administrators understand that technology is always social before it is technological, we must assume that their intransigence is a form of conscious resistance.

That idea of the centre they cling to cannot hold. Up to the point of its failure, everything they do will be predicated on the need for control to ensure its survival. Even their efforts to "decentralize" are merely efforts to retain control in changing circumstances.

A distributed system on the other hand, has little need to waste energy in control because the key to its survival is its capacity to learn. To hang on to control in a networked online world, governments are just going to have to learn to get into a new way of doing things ... because there are many communities, even disadvantaged communities, that are busy learning their way around them.

\section{Who do you want to be today?}

To get to a clear view of community networking as radical practice in social change, we also have to think about how the role of the individual in society is changing and how that affects the question of identity.

The responsibilities of an individual acting as a citizen of a nation state are not the same as the responsibilities of an individual acting as a member of a social network. Nation states are rational constructs, not systems. They see the act of membership in terms of mobilization. That is to say, membership is inherently imposed as a condition external to inclusion.

For the individual as a component of a dynamic network, membership is inherently an inward act of self-identification. A degree of individual autonomy is required before a social network can emerge by self-organization.

Online processes will work to mediate the formation and interactions of social networks. They will not work to mobilize the formation and actions of nation states. This is not to say that online social network process opposes the actions of nation states (although the "content" of a particular network may have that objective). They are merely indifferent to such purposes.

Yes, in either condition, individuals may have internalized the belief that they are members. But a nation state orders the internalization of that belief and sustains institutions intended to socialize for that result. In effect, like reading science fiction, participation requires "a willing suspension of disbelief." A network, on the other hand, does not impose a necessity to believe in membership. Either that quality of self-identification is inherent in every individual so connected, or no network emerges.

While many of the individuals now living daily life online in self-organizing social networks may not be fully conscious of what exactly has changed, they do know that you are either "on" the Net or you are not. They easily recognize and dismiss actions to impose a duty on them, or to organize their direction, as antithetical to the structures of social networking they inhabit. They treat the imposition of order, as opposed to the emergence of order, as an obstacle that the network, to survive, must learn its way around.

But, you declaim, doesn't that rejection of authority replace something valuable, a sense of duty, with the rhetoric of self-interest?" No, it does not. A citizen in a democracy has a right to peace, order and good 
government and a corresponding responsibility to say what that is. An individual in a network has an absolute responsibility to act authentically in the representation of themselves. They are responsible for cooperation in relationship, because a network is a non-zero sum game or it is nothing.

The authority of structure inherent in self-organization is that the network exists and nothing else. The social contract of social networks is not defined outside of the fact of relationship. When we are able to make our social networks then every single action in relationship re-negotiates the social contract as our social networks make us.

It is, therefore, more than the "community networking movement" that has died. It is that the community of practice called community networking is the most likely zone of re-socialization to recognize that the constituents of social structure have changed so as to render the very idea of "movements" as irrelevant to individual action on social change.

In daily life online, bye-bye nation state. Bye-bye movements. Hello state of mind.

Being online represents a change in the deep structure of daily life. In that new structure, the practices through which an individual participates in social networks are altered in the direction of increased autonomy to define who they are. Their personas multiply as a function of their connections. The more sociable they are, the greater networking power they gain.

Identity formation is not static. What I am, I continuously learn in the context of evolving interactions with others. In fact, it is not too strong to speculate that consciousness and the informing of identity are one and the same thing.

While it is true that creation of the new disturbs the past, so does memory. Memory is one aspect of consciousness, the point of reference that supplies our way of seeing. Learning occurs dynamically as an entanglement of our present experience with everything we have known up to now. It alters both what we can see, and what we cannot.

As conscious beings, we have an inherent capacity for self-reference. One thing that Internet Protocol does as a "tool" is to externalize that capacity for self-reference. Does that make the "groupness" of a group of highly connected human beings conscious? Maybe. But, if it were, how would we know? When a group learns (i.e. the expressed patterns of behaviour of the group is visibly modified by its experience), then at least one of the functions of consciousness, memory, is present.

Self-determination of identity, or the actions that increase the capacity of an individual or a community to decide what is inside and outside the boundary between self and other, allows for control of the choices of how self relates to the wider world around it. In other words, it ensures that, just as in Internet Protocol, any-to-any connections in the formation of identity can always occur. This is because the node in the social network, the self, can then reconfigure its message router default settings at will.

We are no longer lonely and afraid in a world we never made. We are in as many worlds as we can imagine. There is nothing absolute or complete about identity. Our being in the many worlds we can inhabit dynamically alters the way those worlds feel. We learn continuously within the experience of relationship who we, in the present moment, are. We (in) form our worlds, and they in turn (in)form us.

If perception is reality and reality is perception, what better way to play the game of life than by rules that grow from your direct experience? The person who is connected by reference to Internet Protocol is neither a Stone Age hunter, nor an agrarian farmer, nor a capitalistic worker. That person is a dreamer who finds that the waking state and the dreaming state are the same. We are a play on our own words, a transubstantiation that makes our words flesh for our consumption.

In the Informed Society, the capacity to imagine what might be (the simulation of possible futures) is valued. That society's social networks are not going to agree to being rendered either realistic or unified. Any person who chooses to participate in an open and networked community has both a right to be there and a responsibility to speak, at least towards the maintenance of the internal rules that structure that community. This is a pragmatics of an entirely different order.

We are the authors of our own fate. We express our own stories and then those stories express us. The best stories are the ones that survive. Those are the stories that inform the Informed Society. 


\section{But what's sociability as machines become social agents?}

To get to a clear view of community networking as radical practice in social change, we also have to see how we are re-creating the mechanical extensions of ourselves in our own image by making them behave as if they were social actors.

"Code is the only language that is executable, meaning that it is the first discourse that is materially affective." (Alexander R. Galloway. Protocol: how control exists after decentralization. MIT Press 2004, p244).

If you want an example of the future of machine agents as social actors, consider a recent newspaper ad for the 2005 Cadillac STS. It lists a GPS-based DVD navigation system, computer assisted suspension, and Blue Tooth compatibility. Then it says, "Pray it doesn't become self-aware."

This is more than an anthropomorphic metaphor. The ad is playing to an expectation that the monster lives but that now is the time that it can be owned. And then its owner will control the fear that it commands. I cannot resist pointing out that rule by fear is the antithesis of the cooperative rules that structure self-organizing systems. So the ad's apparent surface appeal to modernity is actually completely reactionary, quite in keeping with the inherent conservatism of the market for Cadillacs.

Our Internet, our Selves? Not quite. Yes, we make our networks and our networks make us. But the "we" in that phrase now includes encoded agents that interact autonomously in the dynamic alteration of culture. Those agents sense, experience and learn from the environment, modifying it in the process, just as "we" do. All of us now inhabit dynamic systems of human-machine interaction. This represents an increase in the complexity of society and the capacity for radical practice in social relation. In effect, the Temporary Autonomous Zones (TAZ) of Hakim Bey are multiplying towards infinity. Those are the spaces into which the practitioners of community networking have disappeared.

Where are the grass roots in such networks and in what do they root? In terms of what we have become, not of what we have been, where are the spaces for addressing social exclusion that are political? What is disruptive in an era of disruption? . so that our technologies of coping with life (as the way we do things) become different?

The tools do what the culture that designed them intended them to do ... we make our networks and our networks make us. The tools make the dynamic and organic nature of cooperative systems explicit. They embed into themselves a recursive and self- referential capacity to have the use of the tool modify the tool. The ultimate beneficiary of that "practice" is an individual in a community of similar individuals whose capacity to benefit from socio-economic and political development has increased as a function of being online.

\section{We're not citizens. We're connected}

How then can we get a clear view of community networking as radical practice in social change when the functions that make society possible are not organized at all? They are distributed.

The mobilization of a movement assumes a role for organizers who intervene from outside of existing social systems in order to achieve changes inside those systems.

Networks self-organize by incorporating into themselves a few simple relational rules. There are no roles in them for mobilizers, organizers, or even enablers. In fact, networks tend to treat attempts at playing such roles as a problem to learn a way around. Networks encode practice as collective memory of how interaction works. When ideas and memories can connect themselves, what need for association to organize persons having a common interest"?

There is a historical context for the time when we reached the take-off point in public access to the Internet. We elders of those tribes that were present at the creation were specialists in organization. And so we applied what we knew - forms of association - to accomplish our goals in the uses of ICTs for community development. That was then. But, even then, the programmers were saying, "It's the Internet, stupid ...why not just connect?" Now, organization as a method for radical practice seems at odds with daily life online. 
As daily life online becomes ordinary, and as capacity to engender community online becomes an ordinary function of daily life, where then is the locus of radical practice? In code? In use? As we all know, the groupwares used to create and sustain community online are far from perfect. But all sorts of people are applying those groupwares to accomplish ordinary collaborative tasks of daily life, without reference to goals for social change.

The communities of practice about community networking contain people with a lifetime of experience in community development and social action. They know that the attempt to co-opt them into "representation" also makes them a more visible target. They know that confrontation with authority is always a win/lose game. They know that you don't organize or mobilize a network. A network selforganizes, informed through its own becoming. You identify with and participate in a network.

Distributed self-organizing systems (ie. systems where the choice of connection for any participating element is freely available) behave as a common. In a common, the "best" long term strategy is to act (to choose) as if it were an iterative non-zero sum game. You cooperate until the other defects, then defect until the other cooperates. Yes, that works as math, but it's also a moral statement.

In the daily experience of end-to-end connectivity, what governs my decisions to connect? What influences my decisions to form and sustain weak and strong ties? The attempt to connect, whether through weak or strong ties, is always a movement towards morality and responsibility in relationship.

The individual is a self-organized system. The community is a self-organized system. But the active and willing participant in the life of community, the responsible person, the integrated self makes no assumptions about the system's capacity to self-organize. To me, this brings humanity, tolerance, and humility with respect to that state of openness. If I am open to my experience and you are open to your experience, then the "we" that occurs through the cooperative [non-zero sum game] sharing of experience emerges more coherently that it would through the noise of prior assumptions.

What has deepened is relationship. In society structured by autonomous individuals and machine-based agents, we gain, should we chose to use it, increased capacity in the expression of the self. We gain what the people of the book would call, "The connection to a human mind and heart that we only experience when we enter into a world that someone else has created..." Except that now, as authors of our fate, we are that someone else. As the novel writers once were, we are now quite able to proliferate many worlds in our own image. We can demand that the authenticity of our worldviews at least be discussed, if not accepted.

We were never going to organize a movement of people who intuitively understand that "P" for protocol in TCP/IP means a redefinition of ethical relationship. The TCP/IP protocols are the result of the action of autonomous agents (including computers!) and comprise a new system of distributed control.

\section{So what?}

Richard Lowenberg recently noted:

"the changing (nature) of community networks (formalized organizations) to community networking (the diverse process by which an increasing number of communities are taking steps to become inter-networked and to apply this technical level to their economic, civic, cultural, educational and political betterment.)."

A community network, as a form of association, and a community online, as a form of augmented social network, are not the same thing. When you are not yet in the Informed Society, you need forms of association to organize or "mobilize" to get you there. When you are there, the Internet (or rather Internet Protocol) becomes ubiquitously available to sustain cooperative action in creating online social spaces where community can emerge. What you then need are better and better groupwares to augment or sustain forms of social networking. The point of community networking, then, is for individuals to act cooperatively to make the Internet "happen" more rapidly in support of the need for community.

Community networkers do not see ICTs as a "problem" that can be "fixed." We consider community networking to be much more than a "tool" for the use of communities. We see it as a pointer toward a new form of social structure. As a working hypothesis, our actions and practices in design are grounded in that assumption. To date, this assumption remains unconventional. 
A distinction can be made between those entities that are organizations that find themselves "in" the Informed Society and those entities beyond organization that are "of" the Informed Society. Community networking as a practice is part of the second group. The first group is formed by the organizations that consider the net only a tool for the management of their organizations, rather than as a social context to which the form of their organization must adapt. Eventually, those entities that are "of' the Informed Society will predominate over those that have arrived "in" it unexpectedly and usually still hope that radical change in their practices is unnecessary.

To illustrate this distinction by example, the hosting agency for WSIS, the ITU, is not an organization that is of the Information Society. It is the oldest international organization born as a consequence of, and in response to, the postal and telecom technologies of the industrial era. It is an organization trying to survive "in" the Information Society as it emerges. So too are all of the rest of the UN's organizations. By contrast, the Internet Society is a network disguised as an organization that is of the Information Society. And the conflict between organizations like the ITU and networks like ISOC is just starting.

There are critical elements of an understanding of the nature of an Informed Society that are not addressed within the context of WSIS, even in the Civil Society position. A vision of access to open systems, as the basis for open knowledge sharing, open decision making, open values creation, is the key basis for the achievement of social justice.

The Informed Society should be seen as a society of learning communities that self-organize and "internetwork" into open patterns of local-global relationships. The fractal shapes of its economic and ecological zones emerge out of the complexities of its networks. The word that describes the patterns of interaction made by such zones is not "boundaries;" rather it is "flow." Based on that understanding, there are four structural principles shaping the social networks of a Learning Society and the realities of daily life online:

1. The importance, and the absence, of the role of "community" as an essential element of public policy. In community, it is a person's authentic response to the situation, not their professional authority that contributes to the social glue of trust.

2. The importance of self-organization in dynamic systems as a completely different aspect of governance. The concept of "communities of practice" is at the heart of an understanding of how the dynamics of informing, knowing, and learning shape a political economy of ideas. Governance in self-organizing systems that are open and that learn is different from governance in hierarchical systems that control.

3. The absolute need to defend (non-negotiable) the Internet as a commons. In essence, the Internet's code layer, the software that runs the servers, links and connections, is in the public domain because it is written in the languages of Open Source Systems. To continue to function as designed, it must stay there.

4. Effective Use beyond the Digital Divide. The challenge with ICTs is not to provide simple and passive "access" to the ICTs. Rather, it is to provide the means by which individuals in their communities can make effective use of these technologies for productive as well as other processes. What they know, that which informs them, governs their capacity to make effective choices both about what they produce and what they consume.

The disparate elements of the community networking movement have trundled off into the specifics of what made them disparate - social justice, academic research on social change, community-based approaches to socio-economic development, writing code for groupware, and the technological challenges of applying new media and communications in actual communities. But that "movement" never was a tree and branch structure. Being of the Internet, and viscerally getting it about self-organized and distributed systems, we all just knew from the beginning that draining energy and resources from the leaves and branches to fatten a trunk at the centre was never going to happen. We know that the scales that shape selforganization are fractal.

The reason that nobody is writing community networking guides any more is that the locus of community-based radical practice for social change has moved to evolving the state of the art of the groupwares available to augment social networks. But, oddly enough considering what we've learned, the question of the uses of those groupwares remains largely unaddressed. Even among the most aware, the communities of practice about community networking, technological determinism still rules! 
Now that we have crossed the desert and are standing on the edge of that promised land of infinite broadband at zero cost, now what?

- Can we keep our blogs afloat, while all around us others are losing theirs?

- Can we cause a flash mob to emerge to carry mock coffins through the streets of New York (Are flash mobs mobilized?)?

- Can we amortize the cost of a community-owned open backbone before a prime communications carrier can yell, "deregulate?"

- Can we be every when and why while being nowhere at all? Because we understand that code is protocol, can we act autonomously in the context of now to resolve the tensions that now has created?

- Can we tell the governors, who still believe that control is something external to systems and imposed upon them, what good government in self-determined and organized systems actually is?

- Can our communities reclaim the development process for themselves?

- Can we embrace connectedness and interaction as fostering diversity... and reject universality and the global?

- Do we know that participation, especially in webs of association, will follow small world theory and power laws?

- Have we looked deeply into what is happening in art?

- If you really need a network and one is not there - how do you get one?

In effect, the mere acceptance of a transition to an Informed Society and an awareness of what that means creates a medium in which the yeast of new open social networks can grow. That involves recognizing certain kinds of skills that the Informed Society values and requires that are not present in the Industrial Society. When parts of community becomes community online, the human catalysts for community formation need to be conscious of a different kind of presence inside the social networks out of which community online emerges.

In a society of autonomous individuals who choose to act so that self-organizing community emerges, radical practice becomes any act that moves community away from the rational and closer to the sacred. The act of connecting changes the mix of connections and therefore changes the context in which connection learns. There is no way into such a process without action. Just do it. As they still say, "Rough consensus and write code."

There is not going to be a cookbook or primer for community networking. We are going to imagine it as we go.

Yes, this essay is mostly questions, even when I sound like I am making a statement. I do not have an answer for any of this yet that fully squares my actions with my convictions. However, to end with a pointer toward a positive direction, here is one small scenario:

When being online increases the capacity for identity to self-organize, the social fabric of daily life online is woven from community. Ideas of governance grounded in the notion of the nation state cannot absorb the fact of community online as an essential structural principle guiding public policy without radical change in their understanding of the nature of authority and control. It is, therefore, unlikely that nation states will accept the necessity of change. However, it is possible that local governments can and will change more rapidly.

And, after all, that's where your communities are. That's where your most intense shared social context is going to be when it's on its home page. As the broadband networked weirdness intensifies, if you don't look after them, face-to-face, who will? I did warn you this was going to be about the politics of small minds. 